\title{
Synthesis and assessment of substituent effect on (E)-1-(4-fluoro-3-methylphenyl)-3-phenylprop-2-en-1-one compounds
}

\author{
S. Balaji ${ }^{1}$, M. Rajarajan ${ }^{1}$, R. Vijayakumar ${ }^{1}$, V. Manikandan ${ }^{1}$, R. Senbagam ${ }^{1}$ \\ G. Vanangamudi ${ }^{1 *}, \mathrm{G}$. Thirunarayanan ${ }^{2}$, \\ ${ }^{1} \mathrm{PG} \&$ Research Department of Chemistry, Government Arts College, C-Mutlur, \\ Chidambaram-608 102, India \\ 2Department of Chemistry, Annamalai University, Annamalainagar-608 002, India
}

*E-mail address: drgvsibi@gmail.com

\begin{abstract}
Keywords: Synthesis, 4-fluoro-3-methyl acetophenone, UV, IR and NMR spectra, Spectral correlation analysis and substituent effect.
\end{abstract}

\begin{abstract}
In the present investigation, a series of substituted (E)-1-(4-fluoro-3-methylphenyl)3-phenylprop-2-en-1-one compounds were synthesized by condensation reaction of 4-fluoro-3methyl acetophenone with substituted benzaldehydes in the presence of alkali. The yields of synthesized compounds are more than $80 \%$. The structures of all the synthesized compounds were confirmed by their physical constants, UV, IR and NMR spectral data. All the observed spectral data of substituted (E)-1-(4-fluoro-3-methylphenyl)-3-phenylprop-2-en-1-one compounds have been correlated with Hammett substituent constants and $F$ and $R$ parameters using single and multilinear regression analyses. From the results of statistical analysis the effects of substituents have been discussed.
\end{abstract}

\section{INTRODUCTION}

Chalcones are 3-phenylprop-2-en-1-one compounds, in which two aromatic rings are linked by a three carbon $\alpha, \beta$-unsaturated carbonyl system. These are abundant in edible plants and are considered to be precursors of flavonoids and isoflavonoids. The compounds with the backbone of chalcones have been reported to possess various properties by which they find many applications in different fields. Molecules possessing such system have relatively low redox potentials and have a greater probability of undergoing electron transfer reactions. Chalcones are one of the major classes of natural products with widespread distribution in fruits, vegetables, spices, tea and soy based foodstuff have been recently subjects of great interest for their interesting pharmacological activities [1].

Many chalcones have been used as agro chemicals and drugs [2-6]. Various methods are available for the synthesis of chalcone, the most convenient method is the one that involves the Crossed-Aldol condensation of equimolar quantities of acetylated aliphatic or aromatic compounds with substituted benzaldehydes in the presence of aqueous alcoholic alkali [7]. Spectroscopic data is useful for study of effect of substituents using Hammett substituent constants, $F$ and $R$ parameters by single and multi-regression analysis. Recently chemists [8-9] has synthesized and studied spectral correlations of some aryl chalcones and they observed satisfactory correlations using spectral data with Hammett substituent constants, $F$ and $R$ parameters. Literature survey shows that there is no information available regarding the study of UV, IR and NMR spectral correlation of substituted (E)-1-(4-fluoro-3-methylphenyl)-3-phenylprop-2-en-1-one compounds. Therefore the authors have taken efforts to synthesis these 3-phenylprop-2-en-1-one compounds 4-fluoro-3methyl acetophenone with various substituted benzaldehydes by condensation reaction. The various spectral data of these 3-phenylprop-2-en-1-one compounds have been utilized for studying the quantitative structure activity relationships through Hammett correlations. 


\section{EXPERIMENTAL}

\section{1. Materials and Methods}

All the chemicals used in this investigation were purchased from Sigma-Aldrich and EMerck chemical companies. Melting points of all synthesized chalcones were determined in open glass capillaries on Mettler FP51 melting point apparatus and are uncorrected. The shimadzu-1650 ultraviolet spectrophotometer was utilized for recording the absorption maxima $\left(\lambda_{\max }, \mathrm{nm}\right)$, of all chalcones in spectral grade methanol. Infrared spectra $\left(\mathrm{KBr}, 4000-400 \mathrm{~cm}^{1}\right)$ of all chalcones were recorded in avatar-300 Fourier transform spectrophotometer. The NMR spectra of all chalcones were recorded in Bruker AV400 NMR spectrometer, operating $400 \mathrm{MHz}$ and $500 \mathrm{MHz}$ for ${ }^{1} \mathrm{H}$ and $100 \mathrm{MHz}$ for ${ }^{13} \mathrm{C}$ spectra in $\mathrm{CDCl} 3$ solvent using TMS as internal standard.

2. 2. General procedure for crossed-aldol condensation of (E)-1-(4-fluoro-3-methylphenyl)-3phenylprop-2-en-1-one compounds:

A mixture of 4-fluoro-3-methyl acetophenone $(0.05 \mathrm{~mol})$ and various substituted benzaldehydes $(0.05 \mathrm{~mol})$ was dissolved in $50 \mathrm{ml}$ absolute ethanol in a $250 \mathrm{ml}$ round-bottom flask equipped with a magnetic stirrer. Then $50 \mathrm{ml} \mathrm{NaOH}$ solution $\left(1 \mathrm{~g}\right.$ in $\left.50 \mathrm{ml} \mathrm{H}_{2} \mathrm{O}\right)$ was added drop wise to the reaction mixture on vigorous stirring for 30 minutes when solution became turbid. The reaction mixture was neutralized by $0.1 \mathrm{~N} \mathrm{HCl}$ by the precipitation occurred [10-11]. On filtering off, the crude chalcone was dried in air and recrystallized using ethanol to get glittering color solid, and their melting points were observed. The general reaction is shown in Scheme-1. The physical constants are presented in Table-1. The UV, IR and NMR spectral data of these chalcones are presented in Table-2.<smiles>CC(=O)c1ccc(F)c(C)c1</smiles><smiles>[X]c1ccc(C=O)cc1</smiles><smiles>[X]c1ccc(/C=C/C(=O)c2ccc(F)c(C)c2)cc1</smiles>

$\mathrm{X}=\mathrm{H}, 3-\mathrm{Br}, 4-\mathrm{Br}, 2-\mathrm{Cl}, 3-\mathrm{Cl}, 4-\mathrm{Cl}, 3-\mathrm{F}, 4-\mathrm{F}, 4-\mathrm{OCH}_{3}, 4-\mathrm{CH}_{3}, 3-\mathrm{NO}_{2}$

Scheme 1. Synthesis of substituted (E)-1-(4-fluoro-3-methylphenyl)-3-phenylprop-2-en-1-one.

Table 1. Physical constants of substituted (E)-1-(4-fluoro-3-methylphenyl)-3-phenylprop-2-en- 1one compounds.

\begin{tabular}{|c|c|c|c|c|c|}
\hline Entry & $\mathbf{X}$ & $\mathbf{M} . \mathbf{F}$. & M. W. & Yield (\%) & m.p. $\left({ }^{\circ} \mathbf{C}\right)$ \\
\hline 1 & $\mathrm{H}$ & $\mathrm{C}_{16} \mathrm{H}_{13} \mathrm{FO}$ & 240.27 & 86 & $58-60$ \\
\hline 2 & $3-\mathrm{Br}$ & $\mathrm{C}_{16} \mathrm{H}_{12} \mathrm{FBrO}$ & 319.17 & 81 & $57-59$ \\
\hline 3 & $4-\mathrm{Br}$ & $\mathrm{C}_{16} \mathrm{H}_{12} \mathrm{FBrO}$ & 319.17 & 90 & $91-93$ \\
\hline 4 & $2-\mathrm{Cl}$ & $\mathrm{C}_{16} \mathrm{H}_{12} \mathrm{FCl} \mathrm{O}$ & 274.72 & 83 & $60-62$ \\
\hline 5 & $3-\mathrm{Cl}$ & $\mathrm{C}_{16} \mathrm{H}_{12} \mathrm{FCl} \mathrm{O}$ & 274.72 & 92 & $61-63$ \\
\hline 6 & $4-\mathrm{Cl}$ & $\mathrm{C}_{16} \mathrm{H}_{12} \mathrm{FCl} \mathrm{O}$ & 274.72 & 93 & $99-101$ \\
\hline
\end{tabular}




\begin{tabular}{|c|c|c|c|c|c|}
\hline 7 & $3-\mathrm{F}$ & $\mathrm{C}_{16} \mathrm{H}_{12} \mathrm{~F}_{2} \mathrm{O}$ & 258.26 & 82 & $80-82$ \\
\hline 8 & $4-\mathrm{F}$ & $\mathrm{C}_{16} \mathrm{H}_{12} \mathrm{~F}_{2} \mathrm{O}$ & 258.26 & 94 & $88-91$ \\
\hline 9 & $4-\mathrm{OCH}_{3}$ & $\mathrm{C}_{17} \mathrm{H}_{15} \mathrm{FO}_{2}$ & 270.30 & 91 & $49-51$ \\
\hline 10 & $4-\mathrm{CH}_{3}$ & $\mathrm{C}_{17} \mathrm{H}_{15} \mathrm{FO}$ & 254.30 & 82 & $97-99$ \\
\hline 11 & $3-\mathrm{NO} 2$ & $\mathrm{C}_{16} \mathrm{H}_{12} \mathrm{FNO}_{3}$ & 285.27 & 85 & $121-123$ \\
\hline
\end{tabular}

Table 2. The ultraviolet absorption maxima $\left(\lambda_{\max }, \mathrm{nm}\right)$, infrared absorptions $\left(v, \mathrm{~cm}^{-1}\right)$ and NMR chemical shifts $(\delta, \mathrm{ppm})$ spectral data of substituted $(E)-1$-(4-fluoro-3-methylphenyl)-3-phenylprop2-en-1-one compounds.

\begin{tabular}{|c|c|c|c|c|c|c|c|c|c|c|c|c|c|}
\hline \multirow{2}{*}{ Entry } & \multirow{2}{*}{ X } & \multirow{2}{*}{$\lambda_{\max }$} & $v \mathrm{CO}$ & $v \mathrm{CO}$ & \multirow{2}{*}{$v \mathrm{CH}_{\mathrm{ip}}$} & \multirow{2}{*}{$v \mathrm{CH}_{\mathrm{op}}$} & \multirow{2}{*}{$v \mathrm{CH}=\mathrm{CH}_{\mathrm{op}}$} & \multirow{2}{*}{$v \mathrm{C}=\mathrm{C}_{\mathrm{op}}$} & \multirow{2}{*}{$\delta \mathrm{H}_{\alpha}$} & \multirow{2}{*}{$\delta \mathrm{H}_{\beta}$} & \multirow{2}{*}{$\delta \mathrm{C}_{\alpha}$} & \multirow{2}{*}{$\delta \mathrm{C}_{\beta}$} & \multirow{2}{*}{$\delta \mathrm{CO}$} \\
\hline & & & $(s-c i s)$ & $(s$-trans) & & & & & & & & & \\
\hline 1 & $\mathrm{H}$ & 309.0 & 1662.64 & 1597.06 & 1244.09 & 761.88 & 1051.20 & 557.43 & 7.523 & 7.822 & 121.71 & 144.78 & 189.02 \\
\hline 2 & $3-\mathrm{Br}$ & 301.5 & 1658.78 & 1600.92 & 1240.23 & 783.10 & 1060.85 & 538.14 & 7.490 & 7.705 & 122.89 & 142.83 & 188.47 \\
\hline 3 & $4-\mathrm{Br}$ & 315.0 & 1662.64 & 1595.13 & 1242.16 & 812.03 & 1047.35 & 530.42 & 7.508 & 7.747 & 122.15 & 143.32 & 188.73 \\
\hline 4 & $2-\mathrm{Cl}$ & 302.5 & 1664.57 & 1593.20 & 1244.09 & 817.82 & 1043.49 & 551.64 & 7.467 & 8.182 & 124.59 & 140.60 & 189.03 \\
\hline 5 & $3-\mathrm{Cl}$ & 306.0 & 1666.50 & 1597.06 & 1244.09 & 792.74 & 1051.20 & 567.07 & 7.505 & 7.727 & 122.85 & 142.97 & 188.53 \\
\hline 6 & $4-\mathrm{Cl}$ & 312.5 & 1664.57 & 1597.06 & 1244.09 & 819.75 & 1047.35 & 553.57 & 7.485 & 7.750 & 122.06 & 143.25 & 188.69 \\
\hline 7 & $3-F$ & 303.0 & 1660.71 & 1591.27 & 1242.16 & 815.89 & 1049.28 & 551.64 & 7.497 & 7.749 & 122.84 & 143.18 & 188.61 \\
\hline 8 & $4-\mathrm{F}$ & 310.5 & 1664.57 & 1595.13 & 1236.37 & 823.60 & 1049.28 & 565.14 & 7.447 & 7.782 & 121.45 & 143.43 & 188.80 \\
\hline 9 & $4-\mathrm{OCH}_{3}$ & 326.0 & 1664.57 & 1597.06 & 1247.94 & 825.53 & 1043.49 & 551.64 & 7.370 & 7.763 & 119.34 & 144.63 & 189.04 \\
\hline 10 & 4- $\mathrm{CH}_{3}$ & 324.5 & 1658.78 & 1597.06 & 1238.30 & 813.96 & 1045.42 & 555.50 & 7.481 & 7.807 & 120.74 & 144.91 & 189.19 \\
\hline 11 & $3-\mathrm{NO}_{2}$ & 301.0 & 1666.50 & 1598.99 & 1246.02 & 810.10 & 1047.35 & 542.00 & 7.117 & 7.847 & 122.27 & 141.60 & 188.18 \\
\hline
\end{tabular}


Table 3. Results of statistical analysis of ultraviolet absorption maxima $\left(\lambda_{\max }, \mathrm{nm}\right)$, infrared absorptions $\left(v, \mathrm{~cm}^{-1}\right)$ and NMR chemical shifts $(\delta, \mathrm{ppm})$ of substituted $(E)-1$-(4-fluoro-3methylphenyl)-3-phenylprop-2-en-1-one compounds with Hammett $\sigma, \sigma^{+}, \sigma_{\mathrm{I}}, \sigma_{\mathrm{R}}$ and $F$ and $R$ parameters.

\begin{tabular}{|c|c|c|c|c|c|c|c|}
\hline Frequency & Constants & $\mathbf{R}$ & $I$ & $\rho$ & $\mathbf{s}$ & $\mathbf{n}$ & Correlated derivatives \\
\hline \multirow[t]{6}{*}{$\lambda_{\max } \mathrm{nm}$} & $\sigma$ & 0.983 & 315.11 & -26.17 & 5.05 & 11 & $\mathrm{H}, 3-\mathrm{Br}, 4-\mathrm{Br}, 2-\mathrm{Cl}, 3-\mathrm{Cl}, 4-\mathrm{Cl}, 3-\mathrm{F}, 4-\mathrm{F}, 4-\mathrm{OCH}_{3}, 4-\mathrm{CH}_{3}, 3-\mathrm{NO}_{2}$ \\
\hline & $\sigma^{+}$ & 0.968 & 312.88 & -17.91 & 4.63 & 11 & $\mathrm{H}, 3-\mathrm{Br}, 4-\mathrm{Br}, 2-\mathrm{Cl}, 3-\mathrm{Cl}, 4-\mathrm{Cl}, 3-\mathrm{F}, 4-\mathrm{F}, 4-\mathrm{OCH}_{3}, 4-\mathrm{CH}_{3}, 3-\mathrm{NO}_{2}$ \\
\hline & $\sigma_{\mathrm{I}}$ & 0.906 & 319.68 & -24.47 & 7.28 & 11 & $\mathrm{H}, 3-\mathrm{Br}, 4-\mathrm{Br}, 2-\mathrm{Cl}, 3-\mathrm{Cl}, 4-\mathrm{Cl}, 3-\mathrm{F}, 4-\mathrm{F}, 4-\mathrm{OCH}_{3}, 4-\mathrm{CH}_{3}, 3-\mathrm{NO}_{2}$ \\
\hline & $\sigma_{\mathrm{R}}$ & 0.821 & 308.23 & -9.29 & 9.06 & 11 & $\mathrm{H}, 3-\mathrm{Br}, 4-\mathrm{Br}, 2-\mathrm{Cl}, 3-\mathrm{Cl}, 4-\mathrm{Cl}, 3-\mathrm{F}, 4-\mathrm{F}, 4-\mathrm{OCH}_{3}, 4-\mathrm{CH}_{3}, 3-\mathrm{NO}_{2}$ \\
\hline & $F$ & 0.905 & 318.02 & -18.73 & 7.78 & 11 & $\mathrm{H}, 3-\mathrm{Br}, 4-\mathrm{Br}, 2-\mathrm{Cl}, 3-\mathrm{Cl}, 4-\mathrm{Cl}, 3-\mathrm{F}, 4-\mathrm{F}, 4-\mathrm{OCH}_{3}, 4-\mathrm{CH}_{3}, 3-\mathrm{NO}_{2}$ \\
\hline & $R$ & 0.827 & 307.50 & -10.10 & 8.91 & 11 & $\mathrm{H}, 3-\mathrm{Br}, 4-\mathrm{Br}, 2-\mathrm{Cl}, 3-\mathrm{Cl}, 4-\mathrm{Cl}, 3-\mathrm{F}, 4-\mathrm{F}, 4-\mathrm{OCH}_{3}, 4-\mathrm{CH}_{3}, 3-\mathrm{NO}_{2}$ \\
\hline \multirow[t]{6}{*}{$\mathrm{vCO}_{\text {s-cis }}\left(\mathrm{cm}^{-1}\right)$} & $\sigma$ & 0.821 & 1662.68 & 2.54 & 2.78 & 11 & $\mathrm{H}, 3-\mathrm{Br}, 4-\mathrm{Br}, 2-\mathrm{Cl}, 3-\mathrm{Cl}, 4-\mathrm{Cl}, 3-\mathrm{F}, 4-\mathrm{F}, 4-\mathrm{OCH}_{3}, 4-\mathrm{CH}_{3}, 3-\mathrm{NO}_{2}$ \\
\hline & $\sigma^{+}$ & 0.812 & 1663.00 & 1.03 & 2.85 & 11 & $\mathrm{H}, 3-\mathrm{Br}, 4-\mathrm{Br}, 2-\mathrm{Cl}, 3-\mathrm{Cl}, 4-\mathrm{Cl}, 3-\mathrm{F}, 4-\mathrm{F}, 4-\mathrm{OCH}_{3}, 4-\mathrm{CH}_{3}, 3-\mathrm{NO}_{2}$ \\
\hline & $\sigma_{\mathrm{I}}$ & 0.844 & 1661.04 & 5.44 & 2.59 & 11 & $\mathrm{H}, 3-\mathrm{Br}, 4-\mathrm{Br}, 2-\mathrm{Cl}, 3-\mathrm{Cl}, 4-\mathrm{Cl}, 3-\mathrm{F}, 4-\mathrm{F}, 4-\mathrm{OCH}_{3}, 4-\mathrm{CH}_{3}, 3-\mathrm{NO}_{2}$ \\
\hline & $\sigma_{\mathrm{R}}$ & 0.808 & 1663.39 & 1.12 & 2.88 & 11 & $\mathrm{H}, 3-\mathrm{Br}, 4-\mathrm{Br}, 2-\mathrm{Cl}, 3-\mathrm{Cl}, 4-\mathrm{Cl}, 3-\mathrm{F}, 4-\mathrm{F}, 4-\mathrm{OCH}_{3}, 4-\mathrm{CH}_{3}, 3-\mathrm{NO}_{2}$ \\
\hline & $F$ & 0.818 & 1661.85 & 3.11 & 2.76 & 11 & $\mathrm{H}, 3-\mathrm{Br}, 4-\mathrm{Br}, 2-\mathrm{Cl}, 3-\mathrm{Cl}, 4-\mathrm{Cl}, 3-\mathrm{F}, 4-\mathrm{F}, 4-\mathrm{OCH}_{3}, 4-\mathrm{CH}_{3}, 3-\mathrm{NO}_{2}$ \\
\hline & $R$ & 0.814 & 1663.59 & 1.65 & 2.86 & 11 & $\mathrm{H}, 3-\mathrm{Br}, 4-\mathrm{Br}, 2-\mathrm{Cl}, 3-\mathrm{Cl}, 4-\mathrm{Cl}, 3-\mathrm{F}, 4-\mathrm{F}, 4-\mathrm{OCH}_{3}, 4-\mathrm{CH}_{3}, 3-\mathrm{NO}_{2}$ \\
\hline \multirow[t]{6}{*}{$\mathrm{vCO}_{\text {s-trans }}\left(\mathrm{cm}^{-1}\right)$} & $\sigma$ & 0.814 & 1596.09 & 1.36 & 2.74 & 11 & $\mathrm{H}, 3-\mathrm{Br}, 4-\mathrm{Br}, 2-\mathrm{Cl}, 3-\mathrm{Cl}, 4-\mathrm{Cl}, 3-\mathrm{F}, 4-\mathrm{F}, 4-\mathrm{OCH}_{3}, 4-\mathrm{CH}_{3}, 3-\mathrm{NO}_{2}$ \\
\hline & $\sigma^{+}$ & 0.802 & 1596.38 & -0.13 & 2.76 & 11 & $\mathrm{H}, 3-\mathrm{Br}, 4-\mathrm{Br}, 2-\mathrm{Cl}, 3-\mathrm{Cl}, 4-\mathrm{Cl}, 3-\mathrm{F}, 4-\mathrm{F}, 4-\mathrm{OCH}_{3}, 4-\mathrm{CH}_{3}, 3-\mathrm{NO}_{2}$ \\
\hline & $\sigma_{\mathrm{I}}$ & 0.813 & 1596.99 & -1.64 & 2.74 & 11 & $\mathrm{H}, 3-\mathrm{Br}, 4-\mathrm{Br}, 2-\mathrm{Cl}, 3-\mathrm{Cl}, 4-\mathrm{Cl}, 3-\mathrm{F}, 4-\mathrm{F}, 4-\mathrm{OCH}_{3}, 4-\mathrm{CH}_{3}, 3-\mathrm{NO}_{2}$ \\
\hline & $\sigma_{\mathrm{R}}$ & 0.806 & 1598.05 & 8.05 & 2.21 & 11 & $\mathrm{H}, 3-\mathrm{Br}, 4-\mathrm{Br}, 2-\mathrm{Cl}, 3-\mathrm{Cl}, 4-\mathrm{Cl}, 3-\mathrm{F}, 4-\mathrm{F}, 4-\mathrm{OCH}_{3}, 4-\mathrm{CH}_{3}, 3-\mathrm{NO}_{2}$ \\
\hline & $F$ & 0.832 & 1597.78 & -3.38 & 2.61 & 11 & $\mathrm{H}, 3-\mathrm{Br}, 4-\mathrm{Br}, 2-\mathrm{Cl}, 3-\mathrm{Cl}, 4-\mathrm{Cl}, 3-\mathrm{F}, 4-\mathrm{F}, 4-\mathrm{OCH}_{3}, 4-\mathrm{CH}_{3}, 3-\mathrm{NO}_{2}$ \\
\hline & $R$ & 0.905 & 1597.90 & 5.94 & 2.33 & 9 & $\mathrm{H}, 4-\mathrm{Br}, 3-\mathrm{Cl}, 4-\mathrm{Cl}, 3-\mathrm{F}, 4-\mathrm{F}, 4-\mathrm{OCH}_{3}, 4-\mathrm{CH}_{3}, 3-\mathrm{NO}_{2}$ \\
\hline \multirow[t]{6}{*}{$v \mathrm{CH}_{i p}\left(\mathrm{~cm}^{-1}\right)$} & $\sigma$ & 0.812 & 1242.40 & 1.48 & 3.50 & 11 & $\mathrm{H}, 3-\mathrm{Br}, 4-\mathrm{Br}, 2-\mathrm{Cl}, 3-\mathrm{Cl}, 4-\mathrm{Cl}, 3-\mathrm{F}, 4-\mathrm{F}, 4-\mathrm{OCH}_{3}, 4-\mathrm{CH}_{3}, 3-\mathrm{NO}_{2}$ \\
\hline & $\sigma^{+}$ & 0.800 & 1242.67 & 0.05 & 3.53 & 11 & $\mathrm{H}, 3-\mathrm{Br}, 4-\mathrm{Br}, 2-\mathrm{Cl}, 3-\mathrm{Cl}, 4-\mathrm{Cl}, 3-\mathrm{F}, 4-\mathrm{F}, 4-\mathrm{OCH}_{3}, 4-\mathrm{CH}_{3}, 3-\mathrm{NO}_{2}$ \\
\hline & $\sigma_{\mathrm{I}}$ & 0.781 & 1242.19 & 1.25 & 3.52 & 11 & $\mathrm{H}, 3-\mathrm{Br}, 4-\mathrm{Br}, 2-\mathrm{Cl}, 3-\mathrm{Cl}, 4-\mathrm{Cl}, 3-\mathrm{F}, 4-\mathrm{F}, 4-\mathrm{OCH}_{3}, 4-\mathrm{CH}_{3}, 3-\mathrm{NO}_{2}$ \\
\hline & $\sigma_{\mathrm{R}}$ & 0.825 & 1243.56 & 4.29 & 3.41 & 11 & $\mathrm{H}, 3-\mathrm{Br}, 4-\mathrm{Br}, 2-\mathrm{Cl}, 3-\mathrm{Cl}, 4-\mathrm{Cl}, 3-\mathrm{F}, 4-\mathrm{F}, 4-\mathrm{OCH}_{3}, 4-\mathrm{CH}_{3}, 3-\mathrm{NO}_{2}$ \\
\hline & $F$ & 0.811 & 1243.34 & -1.55 & 3.50 & 11 & $\mathrm{H}, 3-\mathrm{Br}, 4-\mathrm{Br}, 2-\mathrm{Cl}, 3-\mathrm{Cl}, 4-\mathrm{Cl}, 3-\mathrm{F}, 4-\mathrm{F}, 4-\mathrm{OCH}_{3}, 4-\mathrm{CH}_{3}, 3-\mathrm{NO}_{2}$ \\
\hline & $R$ & 0.827 & 1243.69 & 3.89 & 3.39 & 11 & $\mathrm{H}, 3-\mathrm{Br}, 4-\mathrm{Br}, 2-\mathrm{Cl}, 3-\mathrm{Cl}, 4-\mathrm{Cl}, 3-\mathrm{F}, 4-\mathrm{F}, 4-\mathrm{OCH}_{3}, 4-\mathrm{CH}_{3}, 3-\mathrm{NO}_{2}$ \\
\hline \multirow[t]{6}{*}{$v \mathrm{CH}_{o p}\left(\mathrm{~cm}^{-1}\right)$} & $\sigma$ & 0.811 & 808.97 & -10.68 & 20.49 & 11 & $\mathrm{H}, 3-\mathrm{Br}, 4-\mathrm{Br}, 2-\mathrm{Cl}, 3-\mathrm{Cl}, 4-\mathrm{Cl}, 3-\mathrm{F}, 4-\mathrm{F}, 4-\mathrm{OCH}_{3}, 4-\mathrm{CH}_{3}, 3-\mathrm{NO}_{2}$ \\
\hline & $\sigma^{+}$ & 0.823 & 808.59 & -10.72 & 20.17 & 11 & $\mathrm{H}, 3-\mathrm{Br}, 4-\mathrm{Br}, 2-\mathrm{Cl}, 3-\mathrm{Cl}, 4-\mathrm{Cl}, 3-\mathrm{F}, 4-\mathrm{F}, 4-\mathrm{OCH}_{3}, 4-\mathrm{CH}_{3}, 3-\mathrm{NO}_{2}$ \\
\hline & $\sigma_{\mathrm{I}}$ & 0.831 & 794.08 & 32.98 & 19.24 & 11 & $\mathrm{H}, 3-\mathrm{Br}, 4-\mathrm{Br}, 2-\mathrm{Cl}, 3-\mathrm{Cl}, 4-\mathrm{Cl}, 3-\mathrm{F}, 4-\mathrm{F}, 4-\mathrm{OCH}_{3}, 4-\mathrm{CH}_{3}, 3-\mathrm{NO}_{2}$ \\
\hline & $\sigma_{\mathrm{R}}$ & 0.905 & 796.26 & -52.21 & 17.71 & 11 & $\mathrm{H}, 3-\mathrm{Br}, 4-\mathrm{Br}, 2-\mathrm{Cl}, 3-\mathrm{Cl}, 4-\mathrm{Cl}, 3-\mathrm{F}, 4-\mathrm{F}, 4-\mathrm{OCH}_{3}, 4-\mathrm{CH}_{3}, 3-\mathrm{NO}_{2}$ \\
\hline & $F$ & 0.844 & 792.43 & 34.48 & 18.56 & 11 & $\mathrm{H}, 3-\mathrm{Br}, 4-\mathrm{Br}, 2-\mathrm{Cl}, 3-\mathrm{Cl}, 4-\mathrm{Cl}, 3-\mathrm{F}, 4-\mathrm{F}, 4-\mathrm{OCH}_{3}, 4-\mathrm{CH}_{3}, 3-\mathrm{NO}_{2}$ \\
\hline & $R$ & 0.905 & 795.48 & -44.06 & 17.58 & 11 & $\mathrm{H}, 3-\mathrm{Br}, 4-\mathrm{Br}, 2-\mathrm{Cl}, 3-\mathrm{Cl}, 4-\mathrm{Cl}, 3-\mathrm{F}, 4-\mathrm{F}, 4-\mathrm{OCH}_{3}, 4-\mathrm{CH}_{3}, 3-\mathrm{NO}_{2}$ \\
\hline \multirow[t]{6}{*}{$\begin{array}{c}v \mathrm{CH}=\mathrm{CH}_{o p} \\
\left(\mathrm{~cm}^{-1}\right)\end{array}$} & $\sigma$ & 0.903 & 1047.50 & 6.53 & 4.68 & 10 & $\mathrm{H}, 4-\mathrm{Br}, 2-\mathrm{Cl}, 3-\mathrm{Cl}, 4-\mathrm{Cl}, 3-\mathrm{F}, 4-\mathrm{F}, 4-\mathrm{OCH}_{3}, 4-\mathrm{CH}_{3}, 3-\mathrm{NO}_{2}$ \\
\hline & $\sigma^{+}$ & 0.902 & 1048.15 & 3.90 & 4.75 & 10 & $\mathrm{H}, 4-\mathrm{Br}, 2-\mathrm{Cl}, 3-\mathrm{Cl}, 4-\mathrm{Cl}, 3-\mathrm{F}, 4-\mathrm{F}, 4-\mathrm{OCH}_{3}, 4-\mathrm{CH}_{3}, 3-\mathrm{NO}_{2}$ \\
\hline & $\sigma_{\mathrm{I}}$ & 0.792 & 1047.63 & 2.86 & 5.02 & 11 & $\mathrm{H}, 3-\mathrm{Br}, 4-\mathrm{Br}, 2-\mathrm{Cl}, 3-\mathrm{Cl}, 4-\mathrm{Cl}, 3-\mathrm{F}, 4-\mathrm{F}, 4-\mathrm{OCH}_{3}, 4-\mathrm{CH}_{3}, 3-\mathrm{NO}_{2}$ \\
\hline & $\sigma_{\mathrm{R}}$ & 0.715 & 1049.48 & 3.59 & 5.01 & 11 & $\mathrm{H}, 3-\mathrm{Br}, 4-\mathrm{Br}, 2-\mathrm{Cl}, 3-\mathrm{Cl}, 4-\mathrm{Cl}, 3-\mathrm{F}, 4-\mathrm{F}, 4-\mathrm{OCH}_{3}, 4-\mathrm{CH}_{3}, 3-\mathrm{NO}_{2}$ \\
\hline & $F$ & 0.791 & 1047.85 & 2.12 & 5.03 & 11 & $\mathrm{H}, 3-\mathrm{Br}, 4-\mathrm{Br}, 2-\mathrm{Cl}, 3-\mathrm{Cl}, 4-\mathrm{Cl}, 3-\mathrm{F}, 4-\mathrm{F}, 4-\mathrm{OCH}_{3}, 4-\mathrm{CH}_{3}, 3-\mathrm{NO}_{2}$ \\
\hline & $R$ & 0.812 & 1049.39 & 2.46 & 5.03 & 11 & $\mathrm{H}, 3-\mathrm{Br}, 4-\mathrm{Br}, 2-\mathrm{Cl}, 3-\mathrm{Cl}, 4-\mathrm{Cl}, 3-\mathrm{F}, 4-\mathrm{F}, 4-\mathrm{OCH}_{3}, 4-\mathrm{CH}_{3}, 3-\mathrm{NO}_{2}$ \\
\hline \multirow[t]{4}{*}{$v \mathrm{C}=\mathrm{C}_{o p}\left(\mathrm{~cm}^{-1}\right)$} & $\sigma$ & 0.903 & 553.72 & -12.83 & 10.88 & 9 & $\mathrm{H}, 3-\mathrm{Br}, 2-\mathrm{Cl}, 4-\mathrm{Cl}, 3-\mathrm{F}, 4-\mathrm{F}, 4-\mathrm{OCH}_{3}, 4-\mathrm{CH}_{3}, 3-\mathrm{NO}_{2}$ \\
\hline & $\sigma^{+}$ & 0.903 & 552.67 & -8.99 & 10.80 & 9 & $\mathrm{H}, 3-\mathrm{Br}, 2-\mathrm{Cl}, 4-\mathrm{Cl}, 3-\mathrm{F}, 4-\mathrm{F}, 4-\mathrm{OCH}_{3}, 4-\mathrm{CH}_{3}, 3-\mathrm{NO}_{2}$ \\
\hline & $\sigma_{\mathrm{I}}$ & 0.729 & 556.00 & -12.09 & 11.17 & 11 & $\mathrm{H}, 3-\mathrm{Br}, 4-\mathrm{Br}, 2-\mathrm{Cl}, 3-\mathrm{Cl}, 4-\mathrm{Cl}, 3-\mathrm{F}, 4-\mathrm{F}, 4-\mathrm{OCH}_{3}, 4-\mathrm{CH}_{3}, 3-\mathrm{NO}_{2}$ \\
\hline & $\sigma_{\mathrm{R}}$ & 0.832 & 547.53 & -18.37 & 10.88 & 11 & $\mathrm{H}, 3-\mathrm{Br}, 4-\mathrm{Br}, 2-\mathrm{Cl}, 3-\mathrm{Cl}, 4-\mathrm{Cl}, 3-\mathrm{F}, 4-\mathrm{F}, 4-\mathrm{OCH}_{3}, 4-\mathrm{CH}_{3}, 3-\mathrm{NO}_{2}$ \\
\hline
\end{tabular}




\begin{tabular}{|c|c|c|c|c|c|c|c|}
\hline & $F$ & 0.811 & 553.20 & -4.55 & 11.46 & 11 & $\mathrm{H}, 3-\mathrm{Br}, 4-\mathrm{Br}, 2-\mathrm{Cl}, 3-\mathrm{Cl}, 4-\mathrm{Cl}, 3-\mathrm{F}, 4-\mathrm{F}, 4-\mathrm{OCH}_{3}, 4-\mathrm{CH}_{3}, 3-\mathrm{NO}_{2}$ \\
\hline & $R$ & 0.825 & 548.18 & -11.95 & 11.13 & 11 & $\mathrm{H}, 3-\mathrm{Br}, 4-\mathrm{Br}, 2-\mathrm{Cl}, 3-\mathrm{Cl}, 4-\mathrm{Cl}, 3-\mathrm{F}, 4-\mathrm{F}, 4-\mathrm{OCH}_{3}, 4-\mathrm{CH}_{3}, 3-\mathrm{NO}_{2}$ \\
\hline \multirow[t]{6}{*}{$\delta \mathrm{H}_{\alpha}(\mathrm{ppm})$} & $\sigma$ & 0.824 & 7.477 & -0.174 & 0.110 & 11 & $\mathrm{H}, 3-\mathrm{Br}, 4-\mathrm{Br}, 2-\mathrm{Cl}, 3-\mathrm{Cl}, 4-\mathrm{Cl}, 3-\mathrm{F}, 4-\mathrm{F}, 4-\mathrm{OCH}_{3}, 4-\mathrm{CH}_{3}, 3-\mathrm{NO}_{2}$ \\
\hline & $\sigma^{+}$ & 0.818 & 7.452 & -0.05 & 0.120 & 11 & $\mathrm{H}, 3-\mathrm{Br}, 4-\mathrm{Br}, 2-\mathrm{Cl}, 3-\mathrm{Cl}, 4-\mathrm{Cl}, 3-\mathrm{F}, 4-\mathrm{F}, 4-\mathrm{OCH}_{3}, 4-\mathrm{CH}_{3}, 3-\mathrm{NO}_{2}$ \\
\hline & $\sigma_{\mathrm{I}}$ & 0.837 & 7.521 & -0.196 & 0.113 & 11 & $\mathrm{H}, 3-\mathrm{Br}, 4-\mathrm{Br}, 2-\mathrm{Cl}, 3-\mathrm{Cl}, 4-\mathrm{Cl}, 3-\mathrm{F}, 4-\mathrm{F}, 4-\mathrm{OCH}_{3}, 4-\mathrm{CH}_{3}, 3-\mathrm{NO}_{2}$ \\
\hline & $\sigma_{\mathrm{R}}$ & 0.831 & 7.397 & -0.23 & 0.112 & 11 & $\mathrm{H}, 3-\mathrm{Br}, 4-\mathrm{Br}, 2-\mathrm{Cl}, 3-\mathrm{Cl}, 4-\mathrm{Cl}, 3-\mathrm{F}, 4-\mathrm{F}, 4-\mathrm{OCH}_{3}, 4-\mathrm{CH}_{3}, 3-\mathrm{NO}_{2}$ \\
\hline & $F$ & 0.830 & 7.503 & -0.139 & 0.116 & 11 & $\mathrm{H}, 3-\mathrm{Br}, 4-\mathrm{Br}, 2-\mathrm{Cl}, 3-\mathrm{Cl}, 4-\mathrm{Cl}, 3-\mathrm{F}, 4-\mathrm{F}, 4-\mathrm{OCH}_{3}, 4-\mathrm{CH}_{3}, 3-\mathrm{NO}_{2}$ \\
\hline & $R$ & 0.832 & 7.403 & -0.157 & 0.115 & 11 & $\mathrm{H}, 3-\mathrm{Br}, 4-\mathrm{Br}, 2-\mathrm{Cl}, 3-\mathrm{Cl}, 4-\mathrm{Cl}, 3-\mathrm{F}, 4-\mathrm{F}, 4-\mathrm{OCH}_{3}, 4-\mathrm{CH}_{3}, 3-\mathrm{NO}_{2}$ \\
\hline \multirow[t]{6}{*}{$\delta \mathrm{H}_{\beta}(\mathrm{ppm})$} & $\sigma$ & 0.901 & 7.811 & -0.069 & 0.137 & 10 & $\mathrm{H}, 3-\mathrm{Br}, 4-\mathrm{Br} 3-\mathrm{Cl}, 4-\mathrm{Cl}, 3-\mathrm{F}, 4-\mathrm{F}, 4-\mathrm{OCH}_{3}, 4-\mathrm{CH}_{3}, 3-\mathrm{NO}_{2}$ \\
\hline & $\sigma^{+}$ & 0.801 & 7.793 & 0.028 & 0.138 & 11 & $\mathrm{H}, 3-\mathrm{Br}, 4-\mathrm{Br}, 2-\mathrm{Cl}, 3-\mathrm{Cl}, 4-\mathrm{Cl}, 3-\mathrm{F}, 4-\mathrm{F}, 4-\mathrm{OCH}_{3}, 4-\mathrm{CH}_{3}, 3-\mathrm{NO}_{2}$ \\
\hline & $\sigma_{\mathrm{I}}$ & 0.797 & 7.815 & -0.042 & 0.138 & 11 & $\mathrm{H}, 3-\mathrm{Br}, 4-\mathrm{Br}, 2-\mathrm{Cl}, 3-\mathrm{Cl}, 4-\mathrm{Cl}, 3-\mathrm{F}, 4-\mathrm{F}, 4-\mathrm{OCH}_{3}, 4-\mathrm{CH}_{3}, 3-\mathrm{NO}_{2}$ \\
\hline & $\sigma_{\mathrm{R}}$ & 0.800 & 7.790 & -0.039 & 0.138 & 11 & $\mathrm{H}, 3-\mathrm{Br}, 4-\mathrm{Br}, 2-\mathrm{Cl}, 3-\mathrm{Cl}, 4-\mathrm{Cl}, 3-\mathrm{F}, 4-\mathrm{F}, 4-\mathrm{OCH}_{3}, 4-\mathrm{CH}_{3}, 3-\mathrm{NO}_{2}$ \\
\hline & $F$ & 0.810 & 7.829 & -0.074 & 0.137 & 11 & $\mathrm{H}, 3-\mathrm{Br}, 4-\mathrm{Br}, 2-\mathrm{Cl}, 3-\mathrm{Cl}, 4-\mathrm{Cl}, 3-\mathrm{F}, 4-\mathrm{F}, 4-\mathrm{OCH}_{3}, 4-\mathrm{CH}_{3}, 3-\mathrm{NO}_{2}$ \\
\hline & $R$ & 0.805 & 7.806 & 0.03 & 0.138 & 11 & $\mathrm{H}, 3-\mathrm{Br}, 4-\mathrm{Br}, 2-\mathrm{Cl}, 3-\mathrm{Cl}, 4-\mathrm{Cl}, 3-\mathrm{F}, 4-\mathrm{F}, 4-\mathrm{OCH}_{3}, 4-\mathrm{CH}_{3}, 3-\mathrm{NO}_{2}$ \\
\hline \multirow[t]{6}{*}{$\delta \mathrm{CO}(\mathrm{ppm})$} & $\sigma$ & 0.991 & 188.93 & -0.97 & 0.12 & 11 & $\mathrm{H}, 3-\mathrm{Br}, 4-\mathrm{Br}, 2-\mathrm{Cl}, 3-\mathrm{Cl}, 4-\mathrm{Cl}, 3-\mathrm{F}, 4-\mathrm{F}, 4-\mathrm{OCH}_{3}, 4-\mathrm{CH}_{3}, 3-\mathrm{NO}_{2}$ \\
\hline & $\sigma^{+}$ & 0.972 & 188.83 & -0.51 & 0.21 & 11 & $\mathrm{H}, 3-\mathrm{Br}, 4-\mathrm{Br}, 2-\mathrm{Cl}, 3-\mathrm{Cl}, 4-\mathrm{Cl}, 3-\mathrm{F}, 4-\mathrm{F}, 4-\mathrm{OCH}_{3}, 4-\mathrm{CH}_{3}, 3-\mathrm{NO}_{2}$ \\
\hline & $\sigma_{\mathrm{I}}$ & 0.907 & 188.15 & -1.03 & 0.20 & 11 & $\mathrm{H}, 3-\mathrm{Br}, 4-\mathrm{Br}, 2-\mathrm{Cl}, 3-\mathrm{Cl}, 4-\mathrm{Cl}, 3-\mathrm{F}, 4-\mathrm{F}, 4-\mathrm{OCH}_{3}, 4-\mathrm{CH}_{3}, 3-\mathrm{NO}_{2}$ \\
\hline & $\sigma_{\mathrm{R}}$ & 0.803 & 188.65 & -0.46 & 0.30 & 11 & $\mathrm{H}, 3-\mathrm{Br}, 4-\mathrm{Br}, 2-\mathrm{Cl}, 3-\mathrm{Cl}, 4-\mathrm{Cl}, 3-\mathrm{F}, 4-\mathrm{F}, 4-\mathrm{OCH}_{3}, 4-\mathrm{CH}_{3}, 3-\mathrm{NO}_{2}$ \\
\hline & $F$ & 0.906 & 189.08 & -0.78 & 0.23 & 11 & $\mathrm{H}, 3-\mathrm{Br}, 4-\mathrm{Br}, 2-\mathrm{Cl}, 3-\mathrm{Cl}, 4-\mathrm{Cl}, 3-\mathrm{F}, 4-\mathrm{F}, 4-\mathrm{OCH}_{3}, 4-\mathrm{CH}_{3}, 3-\mathrm{NO}_{2}$ \\
\hline & $R$ & 0.821 & 188.66 & -0.35 & 0.30 & 11 & $\mathrm{H}, 3-\mathrm{Br}, 4-\mathrm{Br}, 2-\mathrm{Cl}, 3-\mathrm{Cl}, 4-\mathrm{Cl}, 3-\mathrm{F}, 4-\mathrm{F}, 4-\mathrm{OCH}_{3}, 4-\mathrm{CH}_{3}, 3-\mathrm{NO}_{2}$ \\
\hline \multirow[t]{6}{*}{$\delta \mathrm{C} \alpha(\mathrm{ppm})$} & $\sigma$ & 0.965 & 121.48 & 3.11 & 1.07 & 10 & $\mathrm{H}, 3-\mathrm{Br}, 4-\mathrm{Br}, 3-\mathrm{Cl}, 4-\mathrm{Cl}, 3-\mathrm{F}, 4-\mathrm{F}, 4-\mathrm{OCH}_{3}, 4-\mathrm{CH}_{3}, 3-\mathrm{NO}_{2}$ \\
\hline & $\sigma^{+}$ & 0.913 & 120.94 & 2.92 & 1.23 & 10 & $\mathrm{H}, 3-\mathrm{Br}, 4-\mathrm{Br}, 3-\mathrm{Cl}, 4-\mathrm{Cl}, 3-\mathrm{F}, 4-\mathrm{F}, 4-\mathrm{OCH}_{3}, 4-\mathrm{CH}_{3}, 3-\mathrm{NO}_{2}$ \\
\hline & $\sigma_{\mathrm{I}}$ & 0.848 & 120.94 & 2.92 & 1.23 & 11 & $\mathrm{H}, 3-\mathrm{Br}, 4-\mathrm{Br}, 2-\mathrm{Cl}, 3-\mathrm{Cl}, 4-\mathrm{Cl}, 3-\mathrm{F}, 4-\mathrm{F}, 4-\mathrm{OCH}_{3}, 4-\mathrm{CH}_{3}, 3-\mathrm{NO}_{2}$ \\
\hline & $\sigma_{\mathrm{R}}$ & 0.814 & 122.27 & 0.96 & 1.40 & 11 & $\mathrm{H}, 3-\mathrm{Br}, 4-\mathrm{Br}, 2-\mathrm{Cl}, 3-\mathrm{Cl}, 4-\mathrm{Cl}, 3-\mathrm{F}, 4-\mathrm{F}, 4-\mathrm{OCH}_{3}, 4-\mathrm{CH}_{3}, 3-\mathrm{NO}_{2}$ \\
\hline & $F$ & 0.835 & 121.29 & 1.85 & 1.32 & 11 & $\mathrm{H}, 3-\mathrm{Br}, 4-\mathrm{Br}, 2-\mathrm{Cl}, 3-\mathrm{Cl}, 4-\mathrm{Cl}, 3-\mathrm{F}, 4-\mathrm{F}, 4-\mathrm{OCH}_{3}, 4-\mathrm{CH}_{3}, 3-\mathrm{NO}_{2}$ \\
\hline & $R$ & 0.825 & 121.45 & 1.44 & 1.36 & 11 & $\mathrm{H}, 3-\mathrm{Br}, 4-\mathrm{Br}, 2-\mathrm{Cl}, 3-\mathrm{Cl}, 4-\mathrm{Cl}, 3-\mathrm{F}, 4-\mathrm{F}, 4-\mathrm{OCH}_{3}, 4-\mathrm{CH}_{3}, 3-\mathrm{NO}_{2}$ \\
\hline \multirow[t]{6}{*}{$\delta \mathrm{C} \beta(\mathrm{ppm})$} & $\sigma$ & 0.907 & 142.85 & -3.30 & 0.96 & 10 & $\mathrm{H}, 3-\mathrm{Br}, 4-\mathrm{Br} 3-\mathrm{Cl}, 4-\mathrm{Cl}, 3-\mathrm{F}, 4-\mathrm{F}, 4-\mathrm{OCH}_{3}, 4-\mathrm{CH}_{3}, 3-\mathrm{NO}_{2}$ \\
\hline & $\sigma^{+}$ & 0.907 & 143.58 & -2.34 & 0.88 & 10 & $\mathrm{H}, 3-\mathrm{Br}, 4-\mathrm{Br} 3-\mathrm{Cl}, 4-\mathrm{Cl}, 3-\mathrm{F}, 4-\mathrm{F}, 4-\mathrm{OCH}_{3}, 4-\mathrm{CH}_{3}, 3-\mathrm{NO}_{2}$ \\
\hline & $\sigma_{\mathrm{I}}$ & 0.907 & 144.92 & -4.35 & 0.92 & 11 & $\mathrm{H}, 3-\mathrm{Br}, 4-\mathrm{Br}, 2-\mathrm{Cl}, 3-\mathrm{Cl}, 4-\mathrm{Cl}, 3-\mathrm{F}, 4-\mathrm{F}, 4-\mathrm{OCH}_{3}, 4-\mathrm{CH}_{3}, 3-\mathrm{NO}_{2}$ \\
\hline & $\sigma_{\mathrm{R}}$ & 0.811 & 143.04 & -0.90 & 1.36 & 11 & $\mathrm{H}, 3-\mathrm{Br}, 4-\mathrm{Br}, 2-\mathrm{Cl}, 3-\mathrm{Cl}, 4-\mathrm{Cl}, 3-\mathrm{F}, 4-\mathrm{F}, 4-\mathrm{OCH}_{3}, 4-\mathrm{CH}_{3}, 3-\mathrm{NO}_{2}$ \\
\hline & $F$ & 0.904 & 144.43 & -2.86 & 1.14 & 10 & $\mathrm{H}, 3-\mathrm{Br}, 4-\mathrm{Br} 3-\mathrm{Cl}, 4-\mathrm{Cl}, 3-\mathrm{F}, 4-\mathrm{F}, 4-\mathrm{OCH}_{3}, 4-\mathrm{CH}_{3}, 3-\mathrm{NO}_{2}$ \\
\hline & $R$ & 0.821 & 142.91 & -1.20 & 1.34 & 11 & $\mathrm{H}, 3-\mathrm{Br}, 4-\mathrm{Br}, 2-\mathrm{Cl}, 3-\mathrm{Cl}, 4-\mathrm{Cl}, 3-\mathrm{F}, 4-\mathrm{F}, 4-\mathrm{OCH}_{3}, 4-\mathrm{CH}_{3}, 3-\mathrm{NO}_{2}$ \\
\hline
\end{tabular}

$\mathrm{r}=$ Correlation co-efficient; $\rho=$ Slope; $\mathrm{I}=$ Intercept; $\mathrm{s}=$ Standard deviation; $\mathrm{n}=$ Number of substituents. 


\section{RESULT AND DISCUSSION}

\section{1. Spectral linearity}

The spectral linearity of chalcones was studied by evaluating the substituent effects [12-13] with respect to various spectral data. The assigned spectral data of all chalcones such as absorption maximum $\lambda_{\max }(\mathrm{nm})$ of carbonyl groups, infrared carbonyl stretches of $\mathrm{vCO}^{-c i s}$ and s-trans, the deformation modes of vinyl part $\mathrm{CH}$ out of plane, in-plane, $\mathrm{CH}=\mathrm{CH}$ and $>\mathrm{C}=\mathrm{C}<$ out of planes $\left(\mathrm{cm}^{-}\right.$ ${ }^{1}$ ), NMR chemical shifts $\delta(\mathrm{ppm})$ of $\mathrm{H}_{\alpha}, \mathrm{H}_{\beta}, \mathrm{C}_{\alpha}, \mathrm{C}_{\beta}, \mathrm{CO}$ are assigned and these data are correlated with various substituent constants.

\subsection{UV spectral study}

The absorption maxima $\left(\lambda_{\max } \mathrm{nm}\right)$ of synthesized substituted (E)-1-(4-fluoro-3methylphenyl)-3-phenylprop-2-en-1-one compounds were assigned and presented in Table 2. These absorption maxima $\left(\lambda_{\max } \mathrm{nm}\right)$ of these chalcones were correlated with Hammett substituent constants [14-20] and $F$ and $R$ parameters [21] using single and multi-linear regression analysis. Hammett correlation involving the group frequencies and absorption maxima, the form of the Hammett equation employed is

$$
\lambda=\rho \sigma+\lambda_{0}
$$

where $\lambda_{0}$ is the frequency for the parent member of the series.

From the Table-3, it is evident that the UV absorption maximum $\lambda_{\max }(\mathrm{nm})$ values of all substituted 3-phenylprop-2-en-1-one compounds, have shown satisfactory correlations with $\sigma$ $(\mathrm{r}=0.983), \sigma^{+}(\mathrm{r}=0.968), \sigma \mathrm{I}(\mathrm{r}=0.906)$ and $\mathrm{F}$ parameter $(\mathrm{r}=0.905)$. However $\mathrm{UV}$ absorption maximum $\lambda_{\max }(\mathrm{nm})$ values of all substituted (E)-1-(4-fluoro-3-methylphenyl)-3-phenylprop-2-en-1one compounds, have shown poor correlations $(\mathrm{r}<0.900)$ with the Hammett substituent constant $\sigma_{\mathrm{R}}$ and $R$ parameter. This is attributed to the weak resonance effect of the substituents for predicting the reactivity on the UV absorption through resonance as per the conjugative structure as shown in Fig.1.

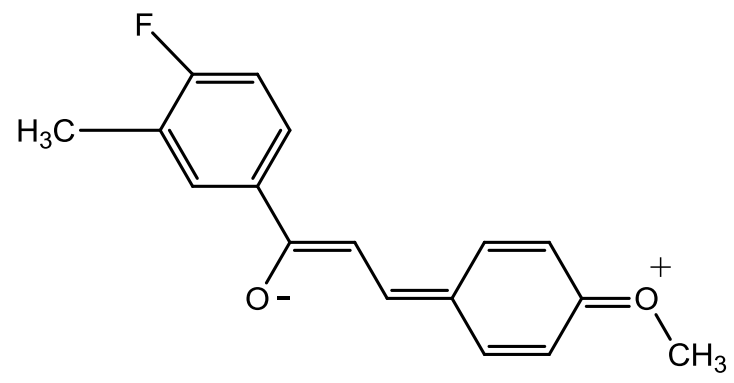

Fig. 1. Resonance conjugative structure

All the correlations have shown negative $\rho$ values. This indicates the operation of reverse substituent effect with respect to UV absorption maximum $\lambda_{\max }(\mathrm{nm})$ values in all substituted 3-phenylprop-2-en-1-one compounds. Since some of the single parameter correlations failed for the UV absorption maximum $\lambda_{\max }(\mathrm{nm})$ values with Hammett constant $\sigma_{\mathrm{R}}$ and $R$ parameter, it is decided to go for multi regression analysis. While seeking the multi regression analysis there is satisfactory correlations are observed as shown in the following equations (2) and (3).

$$
\begin{gathered}
\lambda_{\max }(\mathrm{nm})=317.574( \pm 4.760)-26.741( \pm 9.207) \sigma_{\mathrm{I}}-14.624( \pm 4.867) \sigma_{\mathrm{R}} \\
(R=0.969, \mathrm{n}=11, \mathrm{P}>95 \%) \\
\lambda_{\max }(\mathrm{nm})=315.793( \pm 3.959)-26.832( \pm 8.935) \mathrm{F}-21.678( \pm 7.280) \mathrm{R} \\
(R=0.976, \mathrm{n}=11, \mathrm{P}>95 \%)
\end{gathered}
$$

\section{2. IR spectral study}

The carbonyl stretching frequencies $\left(\mathrm{cm}^{-1}\right)$ of $s$-cis and s-trans conformers are shown in Fig. 2. The IR frequency values are presented in Table 2 . 

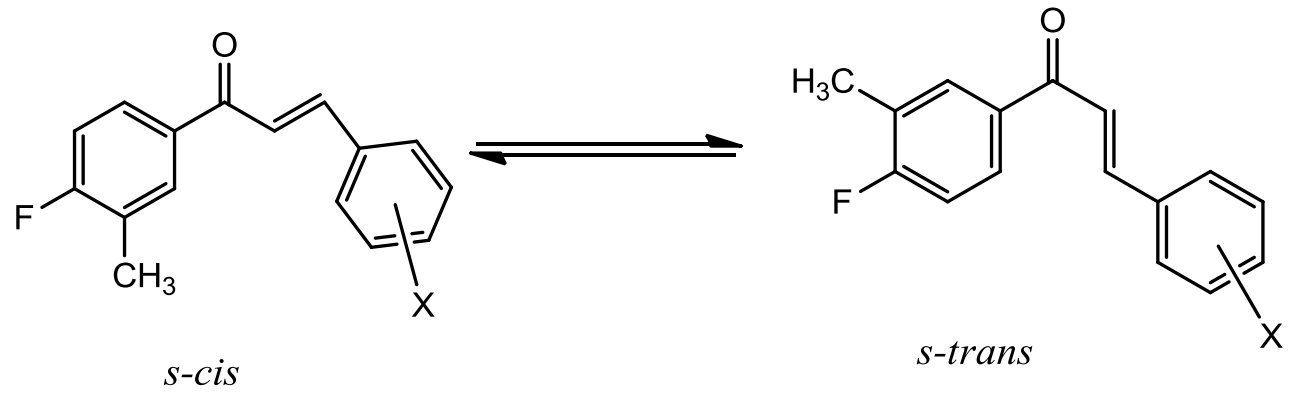

Fig. 2. The $s$-cis and s-trans conformers of (E)-1-(4-fluoro-3-methylphenyl)-3-phenylprop-2-en-1one compounds.

The IR frequency values are correlated with Hammett substituent constants [14-20] and Swain- Lupton's parameters [21]. While seeking Hammett correlation involving group frequencies, the form of the Hammett equation employed is

$$
v=\rho \sigma+v_{0}
$$

where $v_{0}$ is the carbonyl frequencies of substituted system.

\section{2. 1. IR Spectral Correlation of $\mathbf{v C O}$ s-cis $\left(\mathrm{cm}^{-1}\right)$}

The IR frequency $v \mathrm{CO}_{\text {s-cis }}\left(\mathrm{cm}^{-1}\right)$ values of all substituted 3-phenylprop-2-en-1-one compounds have shown poor correlations $(\mathrm{r}<0.900)$ with all Hammett substituent constants and $F$ and $R$ parameters. This is attributed to the weak polar, inductive, field and resonance effects of the substituents All the correlation have shown positive $\rho$ values. This indicates the operation of normal substituent effect with respect to IR frequency $v \mathrm{CO}_{\text {s-trans }}\left(\mathrm{cm}^{-1}\right)$ values in all substituted 3phenylprop-2-en-1-one compounds.

\section{2. 2. IR Spectral Correlation of vCOS-trans $\left(\mathrm{cm}^{-1}\right)$}

The IR frequency $v \mathrm{CO}_{\text {s-trans }}\left(\mathrm{cm}^{-1}\right)$ values of all substituted 3-phenylprop-2-en-1-one compounds except that with $3-\mathrm{Br}$ and $2-\mathrm{Cl}$ substituent have shown satisfactory correlations with $\mathrm{R}$ parameter $(\mathrm{r}=0.905)$. When the substituent that has been given exception is included in regression it reduces the correlations considerably. The IR frequency $v \mathrm{CH}_{I P}\left(\mathrm{~cm}^{-1}\right)$ values of all substituted 3phenylprop-2-en-1-one compounds have shown poor correlations $(\mathrm{r}<0.900)$ with all Hammett substituent constants and $F$ parameter. This is attributed to the weak polar, inductive, resonance and field effects of the substituents. All the correlations except Hammett substituent constants $\sigma+, \sigma \mathrm{I}$ and $\mathrm{F}$ parameter have shown positive $\rho$ values. This indicates the operation of normal substituent effect with respect to IR frequency $v \mathrm{CO}_{\text {s-trans }}\left(\mathrm{cm}^{-1}\right)$ values in all substituted 3-phenylprop-2-en-1one compounds.

\section{2. 3. IR Spectral Correlation of vCH$I P\left(\mathrm{~cm}^{-1}\right)$}

The IR frequency $v \mathrm{CH}_{I P}\left(\mathrm{~cm}^{-1}\right)$ values of all substituted 3-phenylprop-2-en-1-one compounds have shown poor correlations $(\mathrm{r}<0.900)$ with all Hammett substituent constants and $F$ and $R$ parameters. This is attributed to the weak polar, inductive, field and resonance effects of the substituents. All the correlation (except $F$ parameter) have shown positive $\rho$ values. This indicates the operation of normal substituent effect with respect to IR frequency $v \mathrm{CO}_{\text {s-trans }}\left(\mathrm{cm}^{-1}\right)$ values in all substituted 3-phenylprop-2-en-1-one compounds.

\section{2. 4. IR Spectral Correlation of $\mathrm{vCH}_{O P}\left(\mathrm{~cm}^{-1}\right)$}

The IR frequency $v \mathrm{CH}_{O P}\left(\mathrm{~cm}^{-1}\right)$ values of all substituted 3-phenylprop-2-en-1-one compounds, except that with the $\mathrm{H}$ (parant) and 3-Br substituents has shown satisfactory correlations with Hammett substituent constant $\sigma_{\mathrm{R}}(\mathrm{r}=0.905)$. The IR frequency $v \mathrm{CH}_{O P}\left(\mathrm{~cm}^{-1}\right)$ values of all substituted 3-phenylprop-2-en-1-one compounds, except that with the H(parant) substituent has shown satisfactory correlations with $\mathrm{R}$ parameter $(\mathrm{r}=0.905)$. When these substituents that have been 
given exception are included in regression they reduce the correlations considerably. The IR frequency $v \mathrm{CH}_{O P}\left(\mathrm{~cm}^{-1}\right)$ values of all substituted 3-phenylprop-2-en-1-one compounds have shown poor correlations with remaining Hammett substituent constant $\sigma, \sigma^{+}, \sigma_{\mathrm{I}}$, and $F$ perameter. This is attributed to the weak polar, inductive, and field effects of the substituents. All the correlations have shown(except $\sigma_{\mathrm{I}}$, and $F$ perameter ) negative $\rho$ values. This indicates the operation of reverse substituent effect with respect to IR frequency $v \mathrm{CH}_{O P}\left(\mathrm{~cm}^{-1}\right)$ values in all substituted 3-phenylprop2-en-1-one compounds .

\section{2. 5. IR Spectral Correlation of $v \mathrm{CH}=\mathrm{CH}_{O P}\left(\mathrm{~cm}^{-1}\right)$}

The IR frequency $v \mathrm{CH}=\mathrm{CH}_{O P}\left(\mathrm{~cm}^{-1}\right)$ values of all substituted 3-phenylprop-2-en-1-one compounds except that with the 3-Br substituent has shown satisfactory correlations with Hammett substituent constant $\sigma(\mathrm{r}=0.903)$ and $\sigma^{+}(\mathrm{r}=0.902)$. When these substituent that have been given exception are included in regression they reduce the correlations considerably. The IR frequency $v \mathrm{CH}=\mathrm{CH}_{O P}\left(\mathrm{~cm}^{-1}\right)$ values of all substituted 3-phenylprop-2-en-1-one compounds have shown poor correlations with the remaining Hammett substituent constant $\sigma_{\mathrm{R}}, \sigma_{\mathrm{I}}$, and $F$ and $R$ parameters. This is attributed to the weak polar, inductive, field and resonance effects of the substituents. All the correlations (except $\sigma_{R}$ and $\sigma_{I}$ ) have shown positive $\rho$ values. This indicates the operation of normal substituent effect with respect to IR frequency $\mathrm{vCH}=\mathrm{CH}_{O P}\left(\mathrm{~cm}^{-1}\right)$ values in all substituted 3phenylprop-2-en-1-one compounds.

\section{2. 6. IR Spectral Correlation of $v \mathrm{C}=\mathrm{C}_{O P}\left(\mathrm{~cm}^{-1}\right)$}

The IR frequency $v \mathrm{C}=\mathrm{C}_{O P}\left(\mathrm{~cm}^{-1}\right)$ values of all substituted 3-phenylprop-2-en-1-one compounds except 4- $\mathrm{Br}$ and 3-Cl substituents has shown satisfactory correlations with Hammett substituent constants $\sigma(\mathrm{r}=0.903)$ and $\sigma^{+}(\mathrm{r}=0.903)$. When these substituents that have been given exception are included in regression they reduce the correlations considerably. The IR frequency $v \mathrm{C}=\mathrm{C}_{O P}\left(\mathrm{~cm}^{-1}\right)$ values of all substituted 3-phenylprop-2-en-1-one compounds have shown poor correlations with the remaining Hammett substituent constants and $F$ and $R$ parameters. This is attributed to the inductive, field and resonance effects of the substituents. All the correlations have shown negative $\rho$ values. This indicates the operation of reverse substituent effect with respect to IR frequency $\mathrm{vC}=\mathrm{C}_{O P}\left(\mathrm{~cm}^{-1}\right)$ values in all substituted 3-phenylprop-2-en-1-one compounds . Most of the single regression analyses have shown poor correlation, it is decided to go for multi-regression analyses. The multi-regression produced satisfactory correlations with Hammett substituent constants and $F$ and $R$ parameters [21]. The multi correlation equations are given in (5) - (16).

$$
\begin{aligned}
& v \mathrm{CO}_{s-c i s}\left(\mathrm{~cm}^{-1}\right)=1661.37( \pm 1.826)+5.759( \pm 1.917) \sigma_{\mathrm{I}}+2.279( \pm 0.758) \sigma_{\mathrm{R}} \\
& (R=0.946, \mathrm{n}=11, \mathrm{P}>90 \%) \\
& v \mathrm{CO}_{s-c i s}\left(\mathrm{~cm}^{-1}\right)=166.22( \pm 1.743)+4.453( \pm 1.484) \mathrm{F}+3.571( \pm 1.190) \mathrm{R} \\
& (R=0.940, \mathrm{n}=11, \mathrm{P}>90 \%) \\
& v \mathrm{CO}_{\text {s-trans }}\left(\mathrm{cm}^{-1}\right)=1598.148( \pm 1.584)-0.407( \pm 0.135) \sigma_{\mathrm{I}}+7.972( \pm 2.657) \sigma_{\mathrm{R}} \\
& (R=0.960, \mathrm{n}=11, \mathrm{P}>95 \%) \\
& v \mathrm{CO}_{\text {s-trans }}\left(\mathrm{cm}^{-1}\right)=1598.333( \pm 1.525)-1.384( \pm 0.461) \mathrm{F}+5.532( \pm 1.844) \mathrm{R} \\
& (R=0.964, \mathrm{n}=11, \mathrm{P}>95 \%) \\
& v \mathrm{CH}_{i p}\left(\mathrm{~cm}^{-1}\right)=1242.872( \pm 2.424)=1.983( \pm 0.661) \sigma_{\mathrm{I}}+4.692( \pm 1.564) \sigma_{\mathrm{R}} \\
& (R=0.928, \mathrm{n}=11, \mathrm{P}>90 \%) \\
& { } \mathrm{CH}_{i p}\left(\mathrm{~cm}^{-1}\right)=1243.734( \pm 2.240)-0.120( \pm 0.040) \mathrm{F}+3.839( \pm 1.279) \mathrm{R} \\
& (R=0.927, \mathrm{n}=11, \mathrm{P}>90 \%) \\
& v \mathrm{CH}_{o p}\left(\mathrm{~cm}^{-1}\right)=787.298( \pm 11.950)+25.673( \pm 8.557) \sigma_{\mathrm{I}}-47.098( \pm 15.699) \sigma_{\mathrm{R}} \\
& (R=0.959, \quad \mathrm{n}=11, \quad \mathrm{P}>95 \%) \\
& v \mathrm{CH}_{o p}\left(\mathrm{~cm}^{-1}\right)=788.856( \pm 11.060)+21.485( \pm 7.161) \mathrm{F}-34.791( \pm 11.597) \mathrm{R} \\
& (R=0.958, \mathrm{n}=11, \quad \mathrm{P}>95 \%) \\
& v \mathrm{CH}=\mathrm{CH}_{o p}\left(\mathrm{~cm}^{-1}\right)=1048.253( \pm 3.541)+3.529( \pm 1.176) \sigma_{\mathrm{I}}+4.297( \pm 1.432) \sigma_{\mathrm{R}} \\
& (R=0.925, \quad \mathrm{n}=11, \quad \mathrm{P}>90 \%)
\end{aligned}
$$




$$
\begin{aligned}
& v \mathrm{CH}=\mathrm{CH}_{o p}\left(\mathrm{~cm}^{-1}\right)=11048.270( \pm 3.265)+3.637( \pm 1.212) \mathrm{F}+4.038( \pm 1.346) \mathrm{R} \\
& (R=0.923, \quad \mathrm{n}=11, \quad \mathrm{P}>90 \%) \\
& { } \mathrm{C}=\mathrm{C}_{o p}\left(\mathrm{~cm}^{-1}\right)=552.916( \pm 7.365)-15.421( \pm 5.140) \sigma_{\mathrm{I}}-21.451( \pm 7.150) \sigma_{\mathrm{R}} \\
& (R=0.954, \mathrm{n}=11, \mathrm{P}>95 \%) \\
& v \mathrm{C}=\mathrm{C}_{\mathrm{o} p}\left(\mathrm{~cm}^{-1}\right)=551.500( \pm 7.136)-10.749( \pm 3.583) \mathrm{F}-16.592( \pm 5.530) \mathrm{R} \\
& (R=0.935, \mathrm{n}=11, \mathrm{P}>90 \%)
\end{aligned}
$$

\section{3. NMR Spectral study}

In nuclear magnetic resonance spectra, the proton and the ${ }^{13} \mathrm{C}$ chemical shifts $(\delta)$ depends on the electronic environment of the nuclei concerned. The assigned vinyl proton chemical shifts (ppm) have been correlated with reactivity parameters using Hammett equation in the form of

$$
\delta=\delta_{0}+\rho \sigma
$$

where $\delta_{0}$ is the chemical shift of unsubstituted system.

\section{3. 1. ${ }^{1} \mathrm{H}$ NMR Spectral correlation}

\section{3. 2. ${ }^{1} \mathrm{H}$ NMR Spectral Correlations of $\mathbf{H}_{\alpha}(\mathbf{p p m})$}

The assigned $\mathrm{H}_{\alpha}$ chemical shifts $(\delta$, ppm) values are correlated with Hammett constants [1420] and $F$ and $R$ parameters [21], all substituents have shown poor correlation with all Hammett substituent constants and $F$ and $R$ parameters. This is attributed to the weak polar, inductive, resonance and field effects of the substituents. The substituents effect for predicting the reactivity on the chemical shifts through resonance as per the conjugative structure shown in Fig. 1. All the correlations have shown negative $\rho$ values. This indicates the operation of reverse substituent effect.

\section{3. 3. ${ }^{1} \mathrm{H}$ NMR Spectral Correlations of $\mathrm{H}_{\beta}(\mathrm{ppm})$}

The assigned $\mathrm{H}_{\beta}$ chemical shifts $(\delta, \mathrm{ppm})$ values are correlated with Hammett constants and $F$ and $R$ parameters, except 2-Cl substituent have shown satisfactory correlation with Hammett substituent Constant $\sigma(\mathrm{r}=0.901)$. When the substituent that has been given exception is included in regression it reduces the correlations considerably. The IR frequency $v \mathrm{C}=\mathrm{C}_{O P}\left(\mathrm{~cm}^{-1}\right)$ values of all substituted 3-phenylprop-2-en-1-one compounds have shown poor correlations with the remaining Hammett substituent constants and $F$ and $R$ parameters. This is attributed to the polar effects, inductive effects, resonance effects and field effect. All the correlations except $\sigma^{+}$and $R$ parameter have shown negative $\rho$ values. This indicates the operation of reverse substituent effect.

Some of the single regression analyses have shown poor correlations with Hammett substituent constants and swain-Lupton's parameters [21]. It is decided to go for multi-regression, the multi-regression analysis produced satisfactory correlations with the chemical shifts of $(\delta, \mathrm{ppm})$ $\mathrm{H}_{\alpha}$ and $\mathrm{H}_{\beta}$. The multi-correlation equations are given in (18)-(21).

$$
\begin{gathered}
\delta \mathrm{H}_{\alpha}(\mathrm{ppm})=7.481( \pm 0.070)-0.238( \pm 0.793) \sigma_{\mathrm{I}}-0.277( \pm 0.092) \sigma_{\mathrm{R}} \\
(R=0.955, \mathrm{n}=11, \mathrm{P}>95 \%) \\
\delta \mathrm{H}_{\alpha}(\mathrm{ppm})=7.476( \pm 0.066)-0.237( \pm 0.079) \mathrm{F}-0.260( \pm 0.086) \mathrm{R} \\
(R=0.957, \mathrm{n}=11, \mathrm{P}>95 \%) \\
\delta \mathrm{H}_{\beta}(\mathrm{ppm})=7.807( \pm 0.098)-0.050( \pm 0.016) \sigma_{\mathrm{I}}-0.049( \pm 0.016) \sigma_{\mathrm{R}} \\
(R=0.910, \mathrm{n}=11, \mathrm{P}>90 \%) \\
\delta \mathrm{H}_{\beta}(\mathrm{ppm})=7.829( \pm 0.090)-0.074( \pm 0.024) \mathrm{F}-0.001( \pm 0.0003) \mathrm{R} \\
(R=0.914, \mathrm{n}=11, \mathrm{P}>90 \%)
\end{gathered}
$$

\section{4. ${ }^{13} \mathrm{C}$ NMR spectral correlation}

\section{4. 1. ${ }^{13} \mathrm{C}$ NMR spectral correlation of $\delta C_{a}$ carbon}

The assigned $\mathrm{C}_{\alpha}$ chemical shifts $(\delta, \mathrm{ppm})$ correlated with Hammett constants [14-20] and $F$ and $R$ parameters, except that with $2-\mathrm{Cl}$ substituent have shown satisfactory correlation with Hammett substituent constant $\sigma(\mathrm{r}=0.965) \sigma^{+}(\mathrm{r}=0.913)$. When the substituent that has been given exception is included in regression it reduces the correlations considerably. The remaining few 
Hammett substituent constants $\sigma_{\mathrm{I}}, \sigma_{\mathrm{R}}$ and $F$ and $R$ parameters have shown poor correlations $(\mathrm{r}<$ 0.900). This is attributed to the inductive and resonance effects of the substituent. All the correlations have shown positive $\rho$ values. This indicates the operation of normal substituent effect.

\section{4. 2. ${ }^{13} \mathrm{C}$ NMR spectral correlation of $\delta C_{\beta}$ carbon}

The assigned $\mathrm{C}_{\beta}$ chemical shifts $(\delta, \mathrm{ppm})$ correlated with Hammett constants and $F$ and $R$ parameters, All substituent have shown satisfactory correlation with Hammett substituent constant $\sigma_{\mathrm{I}}(\mathrm{r}=0.907) . \mathrm{C}_{\beta}$ chemical shifts $(\delta, \mathrm{ppm})$ correlated with Hammett constants and $F$ and $R$ parameters, expect that with 2-Cl substituent have shown satisfactory correlation with Hammett substituent constant $\sigma(\mathrm{r}=0.907), \sigma^{+}(\mathrm{r}=0.907)$ and $F(\mathrm{r}=0.904)$ parameter. When the substituent that has been given exception is included in regression it reduces the correlations considerably. The remaining one Hammett substituent constants $\sigma_{\mathrm{R}}$ and $R$ parameters [21] have shown poor correlations $(\mathrm{r}<0.900)$. This is attributed to the resonance effects of the substituent. All the correlations have shown negative $\rho$ values. This indicates the operation of reverse substituent effect.

\section{4. 3. ${ }^{13} \mathrm{C}$ NMR spectral correlation of $\delta \mathrm{CO}$ carbon}

The assigned $\mathrm{CO}$ chemical shifts $(\delta, \mathrm{ppm})$ correlated with Hammett constants and $F$ and $R$ parameters, all the substituent have shown satisfactory correlation with Hammett substituent constants $\sigma(\mathrm{r}=0.991), \sigma^{+}(\mathrm{r}=0.972), \sigma_{\mathrm{I}}(\mathrm{r}=0.907) F$ parameter $(\mathrm{r}=0.906)$. When the substituent that has been given exception is included in regression it reduces the correlations considerably. The remaining one Hammett substituent constants $\sigma_{\mathrm{R}}$ and $R$ parameter have shown poor correlations $(\mathrm{r}<$ 0.900). This is attributed to the resonance effect of the substituents. All the correlations have shown negative $\rho$ values. This indicates the operation of reverse substituent effect. Some of the single regression analyses have shown poor correlations with Hammett substituent constants and swainLupton's parameters [21]. It is decided to go for multi-regression, the multi-regression analysis produced satisfactory correlations with the chemical shifts of $(\delta, \mathrm{ppm}) \mathrm{C}_{\alpha}, \mathrm{C}_{\beta}$ and $\mathrm{CO}$. The multi correlation equations are given in (22)-(27).

$$
\begin{gathered}
\delta \mathrm{CO}(\mathrm{ppm})=189.058( \pm 0.105)-1.146( \pm 0.382) \sigma_{\mathrm{I}}-0.694( \pm 0.231) \sigma_{\mathrm{R}} \\
(R=0.988, \mathrm{n}=11, \mathrm{P}>95 \%) \\
\delta \mathrm{CO}(\mathrm{ppm})=189.998( \pm 0.097)-1.086( \pm 0.362) \mathrm{F}-0.818( \pm 0.272) \mathrm{R} \\
(R=0.988, \mathrm{n}=11, \mathrm{P}>95 \%) \\
\delta \mathrm{C}_{\alpha}(\mathrm{ppm})=121.170( \pm 0.854)+3.173( \pm 1.057) \sigma_{\mathrm{I}}+1.601( \pm 0.533) \sigma_{\mathrm{R}} \\
(R=0.953, \mathrm{n}=11, \mathrm{P}>95 \%) \\
\delta \mathrm{C}_{\alpha}(\mathrm{ppm})=121.574( \pm 0.774)+2.856( \pm 0.952) \mathrm{F}+2.678( \pm 0.892) \mathrm{R} \\
(R=0.955, \mathrm{n}=11, \mathrm{P}>95 \%) \\
\delta \mathrm{C}_{\beta}(\mathrm{ppm})=144.662( \pm 0.604)-4.641( \pm 1.547) \sigma_{\mathrm{I}}-1.832( \pm 0.610) \sigma_{\mathrm{R}} \\
(R=0.978, \mathrm{n}=11, \mathrm{P}>95 \%) \\
\delta \mathrm{C}_{\beta}(\mathrm{ppm})=144.135( \pm 0.610)-3.958( \pm 1.330) \mathrm{F}-2.914( \pm 0.971) \mathrm{R} \\
(R=0.974, \mathrm{n}=11, \mathrm{P}>95 \%)
\end{gathered}
$$

\section{CONCLUSION}

A series of ten numbers of substituted (E)-1-(4-fluoro-3-methylphenyl)-3-phenylprop-2-en1-one compounds have been synthesized by condensation of 4-fluoro-3-methyl acetophenone with substituted benzaldehydes. These synthesized substituted (E)-1-(4-fluoro-3-methylphenyl)-3phenylprop-2-en-1-one compounds have been characterized by their physical constants, UV, IR and NMR spectral data. These UV, IR, NMR spectral data of these chalcones has been correlated with Hammett substituent constants, $\mathrm{F}$ and $\mathrm{R}$ parameters. From the results of statistical analyses the effects of substituent on the spectral data have been studied. UV and ${ }^{13} \mathrm{C} N M R$ spectral correlations produced more number of satisfactory correlations. Some of the Hammett substituent constants and swain-Lupton's parameters have shown poor correlation. However, all the multi-regression analyses have shown satisfactory correlations. 


\section{Acknowledgement}

The authors thank DST NMR facility, Department of Chemistry, Annamalai University, Annamalainagar-608 002, for recording NMR spectra of ten synthesized compounds.

\section{References}

[1] Di Carlo G., Mascolo N., Izzo A.A., Capasso F., Life Sci 65 (1999) 122.

[2] Majinda R.R.T., Abegaz B.M., Bezabih M., Ngadjui B.T., Wanjala C.C.W., Mdee L.L., Bojase G., Sialyo A., Masesane I., Yeboah S.O., Pure Appl Chem 73 (2001)1197.

[3] Mirinda C. L., Aponso G. L., Stevens J. F., Denizer M. L., Buhler D. R., Cancer Lett 149 (2000) 21-29.

[4] Monostory K., Tamasi, V., Vereckey, L., Perjesi, P., Toxicology 184 (2003) 203.

[5] Modzelewska A., Pettit C., Achanta G., Davidson N.E., Huang P., Khan S.R., Bioorg Med Chem 14 (2006) 3491.

[6] Sitaram Kumar M., Das J., Iqbal J., Trehan S., Eur J Med Chem 42 (2007) 538.

[7] Thirunarayanan, G., Ananthakrishna Nadar, P., Asian J Chem 14 (2002) 1518.

[8] Thirunarayanan G., Vanangamudi G., E-J. Chem 4 (1) (2007) 90.

[9] Subramanian M., Vanangamudi G., Thirunarayanan G., Spectrochim Acta 110A (2013) 116.

[10] Shendarkar G.R., Savant D.M., Badole K.D., Waghmare G.S., Int. J Phar Tech Res Vol.4, No.3, pp 1129-1135.

[11] GiasuddinAhmed M., Romman U. K. R., Mosaddeq Ahmed S., Kawsari Akhter.,and Ershad Halim Md., Bangladesh J. Sci. Ind. Res. 42(1), 2007, 45-52

[12] Vanangamudi G., Ranganathan K., Thirunaryanan G., World J Chem 7 (2012) 22

[13] Kamalakkannan D., Vanangamudi G., Arulkumaran R., Thirumurthy K., Mayavel P., Thirunarayanan G., Elixir OrgChem 46 (2012) 157.

[14] Sakthinathan S. P., Suresh R., Mala V., Sathiyamoorthi K., Kamalakkannan D., Ranganathan K., Arulkumaran R., Vijayakumar S., Sundararajan R., Vanangamudi G., Thirunarayanan G., Int Lett Chem Phy Astro 6 (2013) 77.

[15] Sekar K. G., Thirunarayanan G., Int Lett Chem Phy Astro 8(2) (2013) 160

[16] Senbagam R., Rajarajan M., Vijayakumar R., Manikandan V., Balaji S., Vanangamudi G., Thirunarayanan G., $W S N 3$ (2015) 155.

[17] Vijayakumar R., Rajarajan M., Balaji S., Manikandan V., Senbagam R., Vanangamudi G.,Thirunarayanan G., $W S N 3$ (2015) 81.

[18] Rajarajan M., Senbagam R., Vijayakumar R., Manikandan V., Balaji S., Vanangamudi G., Thirunarayanan G., $W S N 3$ (2015) 155.

[19] Senbagam R., Rajarajan M., Vijayakumar R., Manikandan., Balaji S., Vanangamudi G., Thirunarayana G., Int Lett Chem Phy Astro 53 (2015) 154.

[20] Thirunarayanan G., Sathiyendiran V., Vanangamudi G., Arulkumaran R., Manikandan V., Suresh R., Kamalakkannan D., Sakthinathan S. P., Sundararajan R., Sathiyamorthi K., Balaji S., Vijayakumar R., Senbagam R. Int Lett Chem Phy Astro50 (2015) 9.

[21] Swain C. G., Lupton E. C. Jr., Am. Chem. Soc 90 (1968) 4328. 